\title{
PERSPECTIVE
}

\section{The definition and classification of glaucoma in prevalence surveys}

\author{
Paul J Foster, Ralf Buhrmann, Harry A Quigley, Gordon J Johnson
}

This review describes a scheme for diagnosis of glaucoma in population based prevalence surveys. Cases are diagnosed on the grounds of both structural and functional evidence of glaucomatous optic neuropathy. The scheme also makes provision for diagnosing glaucoma in eyes with severe visual loss where formal field testing is impractical, and for blind eyes in which the optic disc cannot be seen because of media opacities.

See end of article for authors' affiliations

Correspondence to: Paul J Foster, Department of Epidemiology and International Eye Health, Institute of Ophthalmology, Bath Street, London ECIV 9EL, UK; p.foster@ucl.ac.uk

Accepted for publication 3 August 2001
A $\mathrm{n}$ appropriate case definition is the keystone of epidemiological research whether measuring prevalence, studying risk factors, or conducting clinical trials. This reconsideration of the definition and classification of glaucoma was prompted by our experiences of cross sectional prevalence research in Africa and Asia, and by the difficulty we experienced in identifying and classifying cases and in making valid comparisons with previously published data. The proposed definition of glaucomatous optic neuropathy has evolved from one initially developed for the Kongwa Eye Study in Tanzania. ${ }^{1}$ At the same time, work in Mongolia and Singapore, ${ }^{23}$ where there was a high prevalence of primary angle closure glaucoma (PACG), had prompted a re-examination of the definition of this condition. We were concerned that in previous reports subjects with "latent angle closure glaucoma" had been classified as cases of established glaucoma, despite having normal visual function. This may result in misinterpretation of the estimates of visual morbidity attributable to glaucoma, especially as PACG is believed to be at least as prevalent as primary open angle glaucoma (POAG). ${ }^{4}$

At the biennial congress of the International Society for Geographical and Epidemiological Ophthalmology held in Leeuwenhorst, the Netherlands, in June 1998, a group interested in glaucoma epidemiology met to discuss the prototype system. This has since been discussed further, and various experts in the fields of glaucoma research and clinical practice consulted. (The appendix lists participants and co-opted advisers.) The views presented here are, however, those of the authors. Our aim has been to present a practical framework which can be tested and discussed further.

\section{PROPOSED DEFINING FEATURES OF GLAUCOMA}

The fundamental concept of the proposed classification for cross sectional epidemiological re- search is that the term glaucoma is reserved for people with established, visually significant, end organ damage. In the public health context, glaucoma can be seen as an optic neuropathy associated with characteristic structural damage to the optic nerve and associated visual dysfunction that may be caused by various pathological processes.

\section{Structural damage-optic neuropathy}

The feature that differentiates glaucoma from other causes of visual morbidity is a characteristic pattern of damage to the optic nerve head. This is most easily recognised at the superior and inferior poles of the optic disc. The vertical cup:disc ratio (VCDR) has proved to be a simple, relatively robust index of glaucomatous loss of the neuroretinal rim. As with intraocular pressure, VCDR is a continuous variable within the population.

One approach would be to determine the range of CDR in people with normal visual function (normal visual field) in a population. This group of individuals will therefore be "hypernormal," as those with visual dysfunction due to causes other than glaucoma would be excluded. The choice of where to place the division between "normal" and "abnormal" is, for the time being, arbitrary and partially flawed by the fact that there is overlap between the range of CDRs in those with and without glaucomatous visual loss. Faced with this dilemma, we propose that the statistical convention that a probability of $<5 \%$ representing a significant deviation from normal be invoked. Therefore, the CDR above which $2.5 \%$ of the normal population lie defines the "upper limit of normal" (the other $2.5 \%$ falling below the normal distribution). By using the 97.5 th percentile, one avoids making the assumption that CDR is normally distributed (it has been found to be Gaussian in some studies, but not in others). We also suggest using the 97.5 th percentile value for CDR asymmetry as a second criterion for abnormality. Examples of what these criteria might be for some populations are shown in Table 1.

\section{Functional damage}

While most published definitions of glaucoma include the presence of "characteristic visual field defects," many authors fail to provide quantitative, clearcut descriptions of what this means. The broadly accepted principles are summarised in Table 2 .

\footnotetext{
Abbreviations: PACG, primary angle closure glaucoma; POAG, primary open angle glaucoma; VCDR, vertical cup:disc ratio; IOP, intraocular pressure; PAC, primary angle closure; PAS, peripheral anterior synechiae
} 
Table 1 Vertical cup:disc ratio (VCDR) distribution in people with normal visual fields in one African and three Asian countries

\begin{tabular}{|c|c|c|c|c|c|c|}
\hline & \multicolumn{2}{|l|}{ CDR } & \multicolumn{2}{|c|}{ CDR asymmetry } & \multirow[b]{2}{*}{$\begin{array}{l}\text { Subjects with a } \\
\text { normal field }\end{array}$} & \multirow[b]{2}{*}{$\begin{array}{l}\text { Total number } \\
\text { of subjects }\end{array}$} \\
\hline & $\begin{array}{l}97.5 \text { th } \\
\text { percentile }\end{array}$ & $\begin{array}{l}\text { 99.5th } \\
\text { percentile }\end{array}$ & $\begin{array}{l}97.5 \text { th } \\
\text { percentile }\end{array}$ & $\begin{array}{l}99.5 \text { th } \\
\text { percentile }\end{array}$ & & \\
\hline Bangladesh* & 0.70 & 0.85 & 0.15 & 0.3 & $220 *$ & 2426 \\
\hline Mongolia† & 0.70 & 0.70 & 0.2 & 0.3 & 1551 & 1717 \\
\hline Singapore $†$ & 0.71 & 0.82 & 0.21 & 0.32 & 835 & 1090 \\
\hline Tanzania† & 0.7 & 0.8 & 0.2 & 0.3 & 2524 & 3268 \\
\hline
\end{tabular}

*Field testing was carried out in subjects with either $C D R \geqslant 0.35$ or $I O P \geqslant 18 \mathrm{~mm} \mathrm{Hg}$. Data presented

include those who did not meet these criteria, and therefore did not undergoing field testing.

†Field testing carried out on all subjects.

\begin{tabular}{l} 
Table 2 Characteristics of glaucomatous field \\
defects \\
\hline (1) Asymmetrical across the horizontal midline (in early/moderate \\
cases) \\
(2) Located in the mid-periphery (in early/moderate cases) \\
(3) Clustered in neighbouring test points \\
(4) Reproducible on at least two occasions \\
(5) Not explained by any other disease \\
(6) Considered a valid representation of the subjects functional status \\
(based on performance indices such as false positive rate)
\end{tabular}

These principles fail to account for the possibility of diffuse damage to the visual field in glaucoma. While some diffuse loss clearly must occur, its magnitude and importance in specific glaucoma diagnosis are difficult to determine. ${ }^{5}$ Following consultation with a group of researchers interested in the psychophysics of glaucoma, we have adopted the following as the "gold standard" of glaucomatous visual field loss. The glaucoma hemifield test graded "outside normal limits" and a cluster of three contiguous points at the 5\% level on the pattern deviation plot, using the threshold test strategy with the 24-2 test pattern of the Zeiss-Humphrey field analyser 2. This is not intended to indicate that this device is the only acceptable tool for field analysis. However, we consider it the standard against which others should be validated.

\section{Glaucoma}

The relation between VCDR and proved visual field abnormalities is complex. Some eyes have reproducible visual field defects although they have a CDR that lies within the range defined as normal by the criteria we have selected. Figure 1 shows the relation between CDR and the cumulative probability of a reproducible field defect CDR among Chinese Singaporeans (unpublished data, Paul Foster, Steve Seah, Singapore National Eye Centre, 2001).

We further propose that an individual with field loss who meets the stated criteria (and optic disc meeting criteria for abnormality) in one eye has glaucoma. This takes account of the fact that damage is often present in one eye before the other. However, we appreciate that this monocular based definition may not be representative of a subject's functional capacity. ${ }^{\circ}$

We have not sought to specify that the visual field defect should be "consistent" with the pattern of structural damage to the optic nerve-for example, requiring that inferior field loss must be matched with superior optic disc rim loss. This may lower the specificity of the definition, although we believe the interobserver variation in making this judgment is potentially so great as to introduce greater weakness to the scheme. We therefore suggest that structural features exceeding the specified limits, combined with a field defect that meets the above criteria, will constitute the definition of glaucoma damage.

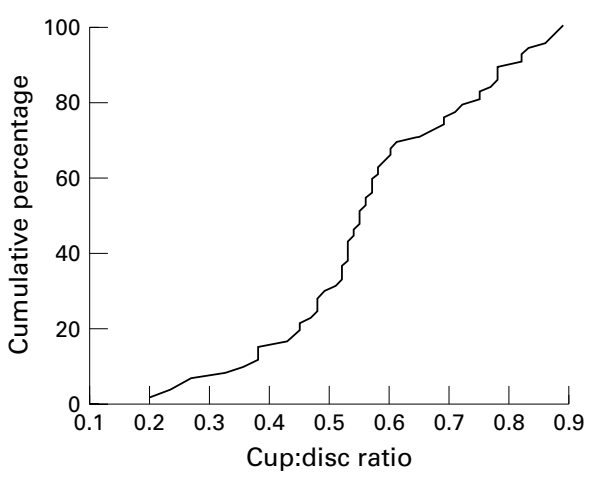

Figure 1 The cumulative percentage of vertical CDR distribution among subjects able to complete visual field testing in a population survey ${ }^{3}$ in whom a reproducible visual field defect Iglaucoma hemifield test "outside normal limits" and a four point cluster $(p<5 \%)$ on the pattern deviation plot) was identified. The data shown here are based on 61 of 67 eyes. Fives eyes were excluded because lens opacity was sufficient to account for the field defect. One eye was excluded because diabetic retinopathy was present. Several eyes with severe visual field loss were not able to produce a reliable visual field test result.

\section{Levels of evidence}

It is therefore envisaged that cases of glaucoma would be classified according to three levels of evidence. The highest level of certainty requires optic disc abnormalities (VCDR $>97.5$ th percentile in the normal population) and visual field defect compatible with glaucoma. In the second, if a visual field test could not be performed satisfactorily, a severely damaged optic disc $(\mathrm{VCDR}>99.5$ th percentile of the normal population) would be sufficient to make the diagnosis. Lastly, if the optic disc could not be examined because of media opacity (and, hence, no field test was also possible), an IOP exceeding the 99.5th percentile of the normal population, or evidence of previous glaucoma filtering surgery, may be taken as sufficient for a diagnosis of glaucoma (see Table 3 for summary).

\section{CLASSIFICATION ACCORDING TO MECHANISM OF DAMAGE \\ POAG and the role of IOP}

Although the level of intraocular pressure (IOP) is one of the most consistent risk factors for the presence of glaucoma, the concept that statistically raised IOP is a defining characteristic for glaucoma has been almost universally discarded. This is based on several population based studies that document the typical disc and field damage of glaucoma in people with a statistically normal IOP and, conversely, people with statistically elevated IOP and no evidence of optic neuropathy. We propose to follow this current convention except for category 3 diagnosis, as detailed above.

POAG is therefore optic nerve damage meeting any of the three categories of evidence above, in an eye which does not 
Table 3 The diagnosis of glaucoma in cross sectional prevalence surveys (The diagnosis is made according to three levels of evidence)

Category 1 diagnosis (structural and functional evidence) Eyes with a CDR or CDR asymmetry $\geqslant 97.5$ th percentile for the normal population, or a neuroretinal rim width reduced to $\leqslant 0.1 \mathrm{CDR}$ (between 11 to 1 o'clock or 5 to $7 o^{\prime}$ clock) that also showed a definite visual field defect consistent with glaucoma.

Category 2 diagnosis (advanced structural damage with unproved field loss)

If the subject could not satisfactorily complete visual field testing but had a CDR or CDR asymmetry $\geqslant 99.5$ th percentile for the normal population, glaucoma was diagnosed solely on the structural evidence.

In diagnosing category 1 or 2 glaucoma, there should be no alternative explanation for CDR findings (dysplastic disc or marked anisometropia) or the visual field defect (retinal vascular disease, macular degeneration, or cerebrovascular disease). Category 3 diagnosis (Optic disc not seen. Field test impossible) If it is not possible to examine the optic disc, glaucoma is diagnosed if: (A) The visual acuity $<3 / 60$ and the IOP $>99.5$ th percentile, or (B) The visual acuity $<3 / 60$ and the eye shows evidence of glaucoma filtering surgery, or medical records were available confirming glaucomatous visual morbidity.

have evidence of angle closure on gonioscopy, and where there is no identifiable secondary cause.

\section{Primary angle closure and narrow drainage angles}

The current classification of PACG is largely based on clinical observations in European derived people, among whom the condition is scarce. While the acute, symptomatic phase is dramatic, it occurs in only a minority of those with PACG diagnosed in population based surveys in African and Asian settings. ${ }^{2378}$ Rather, a chronic, asymptomatic form of PACG predominates. Thus, a full re-evaluation of the definition of this disease is appropriate, with emphasis placed on visual loss rather than symptomatic disease.

We propose that it would be useful to distinguish between the mechanism by which IOP becomes elevated and the resultant damage that is caused by PACG. To do this, people meeting gonioscopic criteria for narrow angles and with evidence of significant obstruction of the functional trabecular meshwork by the peripheral iris would be classified as having primary angle closure (PAC). Those in whom PAC had led to significant glaucomatous damage to the optic nerve would be defined as having PACG. This is not intended to indicate that those with PAC do not require treatment. It is intended to differentiate between those with and without damaged visual function attributable to glaucomatous optic neuropathy. People with PAC and other causes of visual loss, such as iris damage, non-glaucomatous optic atrophy, lens opacity, and corneal endothelial failure should be separately identified.

This approach to classification differs from the scheme found in most textbooks in which people with a narrow drainage angle and either raised IOP or peripheral anterior synechiae (PAS) are said to have primary angle closure "glaucoma." Thus, in this new concept, PAC includes both asymptomatic people with occludable angles who have not had an acute attack, and those with PAC who have had an attack that was treated promptly but suffered no detectable nerve damage. As many as $60-75 \%$ of people suffering an acute, symptomatic episode of angle closure recover without optic disc or visual field damage, ${ }^{90}$ at least in the short term. If one intends the term glaucoma to signify a disease characterised by an irreversible defect in visual function, then many people suffering symptomatic episodes of high IOP or those with narrow drainage angles who are as yet asymptomatic do not meet this criterion for nerve injury. They share anatomical and physiological characteristics with those whose angle closure has led to field loss,
Table 4 Classification of primary angle closure (PAC)

(1) Primary angle closure suspect

An eye in which appositional contact between the peripheral iris and posterior trabecular meshwork is considered possible (see footnote).

(2) Primary angle closure (PAC)

An eye with an occludable drainage angle and features indicating that trabecular obstruction by the peripheral iris has occurred, such as peripheral anterior synechiae, elevated intraocular pressure, iris whorling (distortion of the radially orientated iris fibres), "glaucomfleken" lens opacities, or excessive pigment deposition on the trabecular surface. The optic disc does not have glaucomatous damage.

(3) Primary angle closure glaucoma (PACG)

PAC together with evidence of glaucoma, as defined above.

In epidemiological research this has most often been defined as an angle in which $\geqslant 270^{\circ}$ of the posterior trabecular meshwork (the part which is often pigmented) cannot be seen. This definition is arbitrary and its evaluation in longitudinal study is an important priority. Producing a more evidence based definition of this parameter is a major research priority.

but they deserve to be considered separately for the purposes of the definitions we have intended to construct. This classification scheme is summarised in Table 4.

\section{Glaucoma with secondary ocular pathology}

Not all prevalence studies of glaucoma have separated primary and secondary glaucoma in consistent fashion, if they have done so at all. None the less, the estimated proportion of glaucoma damage that is clearly secondary to other ocular or systemic disease, or to trauma, may represent as much as $20 \%$ of all glaucoma. While we argue above for elimination of IOP as a defining feature of primary OAG or ACG, secondary glaucoma is properly considered to represent those eyes in which a second form of ocular pathology has caused IOP above the normal range, leading to optic nerve damage. We propose that the diagnosis of secondary glaucoma only be based on the presence of optic neuropathy, in so far as it is possible to determine this, in the presence of a second ocular pathological process. These processes may include one of the following:

(1) neovascularisation

(2) uveitic

(3) trauma

(4) lens related.

There are arguments for and against including people with glaucoma and pigment dispersion syndrome or pseudoexfoliation syndrome as cases of secondary glaucoma. They have been omitted from the list above, on the premise that they represent a variant of POAG, although this view remains to be fully vindicated. It must be recognised that many eyes with secondary glaucoma have opaque media, precluding optic disc and visual field examinations. Hence, many of the secondary glaucoma examples will be diagnosed with the category 3 information detailed above, when optic neuropathy is inferred from reduced visual acuity and a relative afferent pupil defect, in the presence of raised IOP. Furthermore, a substantial number of these people are affected unilaterally compared to bilateral involvement in primary glaucoma.

On the other hand, there will be eyes with processes such as pseudoexfoliation or uveitis with IOP above the normal range, but in which the disc is visible and seen to be normal. For consistency, people with eyes with these features will be categorised as secondary ocular hypertensives, or secondary glaucoma suspects.

\section{Glaucoma suspects}

Our categorisation aims to separate an examined population into those who did not have glaucoma, those who had one of the defined forms of glaucoma, and those who had some 
Table 5 Criteria for classification as glaucoma suspect

Disc suspects. Those who met category 1 (but not category 2) disc criteria, but were not proved to have definite field defects.

Field suspects. Those with definite field defects, but not meeting category 1 disc criteria.

Those with optic disc margin haemorrhages.

Those with an IOP $\geqslant 97.5$ th percentile.

Those with an occludable drainage angle, but normal optic disc, visual field, intraocular pressure, and no peripheral anterior synechiae.

suspicion of glaucoma. The various reasons that a person would be considered as a glaucoma suspect are summarised in the Table 5 .

\section{DISCUSSION}

The proposed scheme provides a framework for classifying cases of glaucoma in cross sectional population based research. It places the emphasis of the diagnosis on glaucomatous optic neuropathy with a reproducible visual field defect, but includes criteria for some eyes in which visual field testing or disc evaluation are impossible.

Limiting the use of the term "glaucoma" for those people with established end organ damage - that is, a visually significant optic neuropathy, provides a uniform definition across the various causal mechanisms: primary open angle, primary angle closure, and secondary to other pathology. The "glaucoma as damage" approach is attractive for a number of reasons. Firstly, it is conceptually simple. Secondly, it limits the features required to make the diagnosis to direct measurements of the structure and function of the optic nerve. Thirdly, it is also less arbitrary in specifying divisions between what constitutes normality and disease, because we have considerable information on the distribution of optic nerve structural and functional traits in the population of developed countries.

Potential weaknesses in the use of VCDR as a defining feature for glaucoma include variation in the size of the optic disc between individuals, ${ }^{112}$ variation in the number of axons in the optic nerve, from a minimum of 816000 to a maximum of 1502000 (mean 1159000 plus or minus 196000) in Europeans $^{13}$ and the observation that larger optic nerves have a larger neuroretinal rim area ${ }^{14}$ and contain more axons. ${ }^{15}$ Correction for variation in disc size when assessing the CDR has been suggested. ${ }^{16-18}$

However, the precise VCDR and degree of asymmetry that denote statistical abnormality cannot necessarily be extrapolated to other populations without justification from further data. For example, there is evidence that the disc size and CDR in African-Americans is larger than in European-Americans, although the area of the neuroretinal rim is similar. ${ }^{19-21}$ However, a recent report cited 97.5 th and 99.5 th percentile figures for VCDR distribution in a population in the Netherlands as 0.73 and 0.78 respectively. ${ }^{22}$ These are remarkably similar to those found in Asian populations (Table 1).

The emphasis on end organ damage as the defining characteristic of glaucoma has led to the separation of people with an angle closure into (1) primary angle closure suspects, (2) primary angle closure (where there are signs of disturbed structure or function but no visually significant optic nerve damage), and (3) primary angle closure glaucoma (where optic nerve damage is present). The diagnostic criteria employed in eight major population based studies of glaucoma in areas with a high prevalence of PACG are shown in Table 6. Only three out of eight allowed for optic disc and visual field abnormalities in considering the diagnosis of PACG. In all eight studies, a raised IOP with gonioscopic abnormalities was sufficient to diagnose PACG. The logic of this convention is questionable since natural history data in Europeans suggest that many such people never lose vision. ${ }^{23}$ The Inuit people of Greenland have the highest rate of primary angle closure of any ethnic group. ${ }^{24}$ Follow up over 10 years suggests that even "high risk" individuals within this population have less than a $1 \%$ annual risk of suffering a symptomatic episode or developing ocular damage from angle closure. ${ }^{25}$

Thus, the diagnostic criteria proposed here are likely to lead to alterations in prevalence estimates, particularly for PACG. Since some of those with narrow angles but normal discs will now be classified as PAC, the population with PACG may be lower than in previous surveys that included both in one category. Both are likely to benefit from iridectomy, but the former (PAC) are likely to be cured, while the latter will require more intensive follow up and treatment-indeed much like the treatment for POAG.

Published glaucoma surveys in different populations have varied widely in the availability and sophistication of the instruments available for measurement and diagnosis, in the time available for repeat testing (for example, of a suspect visual field test), and in the money available for an adequate number of examiners and assistants. The optic disc cupping may have been assessed by direct ophthalmoscopy, binocular indirect ophthalmoscopy at the slit lamp, with or without an eye piece graticule, stereoscopic disc photography, a scanning laser ophthalmoscope, or other imaging device. Surveys can thus be arranged in a hierarchy of degrees of completeness and sophistication of the examination.

We do not know the true effect that differences in the methodology may have on the calculated prevalence using the proposed definition. This could be estimated by careful analysis of previous survey data and future glaucoma surveys in which more than one methodological approach is used and comparisons are made.

Considering field testing for the purposes of our definition standards, tests, and instruments other than the Humphrey field analyser (HFA) 2 may be used, but each should be individually validated against this standard. Good equivalence has already been demonstrated for the Zeiss-Humphrey 1 instrument with the newer HFA 2 instrument, and for the SITA testing strategy compared with the standard test algorithm. ${ }^{26}{ }^{27}$

Table 6 Diagnostic criteria for PACG in population based research

\begin{tabular}{llllllll}
\hline Country & Symptoms & IOP & Gonioscopy & Disc damage & Field loss & PT & Acute attack \\
\hline Greenland $^{30}$ & + (with PT or IOP) & + & A & - & - & + & + \\
Alaska $^{31}$ & + & + (with VF) & A & + & + (with lOP) & - & - \\
China $^{32}$ & + & + & + & - & - & - & + \\
Tibet $^{33}$ & + & + & + & - & - & - \\
Japan $^{34}$ & - & + (with gonio) & + (with IOP) & - & - & - & - \\
South Africa $^{7}$ & + & + & A & + (with VF loss) & + (with disc) & - & - \\
Mongolia $^{2}$ & + & + & A & + (with VF loss) & + (with disc) & - & - \\
Taiwan $^{8}$ & - & + & A & - & - & + & + \\
\hline
\end{tabular}

$\mathrm{A}=$ absolute requirement,$+=$ additional feature sufficient for diagnosis combined with absolute requirement, $-=$ not required $/$ not done

$\mathrm{PT}=$ provocative testing, $\mathrm{VF}=$ visual fields, $\mathrm{IOP}=$ intraocular pressure, gonio = findings on gonioscopic examination. 
Reliability of test results is an important consideration. The standards for check trials provided by the Zeiss-Humphrey instrument may not be appropriate. Fixation loss scores may bear little relation to fixation accuracy, ${ }^{28}$ and are especially sensitive to mis-plotting of the blind spot. Furthermore, the precision of false negative and false positive indices is very poor, given the number of test points. The $95 \%$ CIs around a $33 \%$ false negative rate may be from $13 \%$ to $53 \%{ }^{29}$ The software for calculation of false negative and positive responses in the newer HFA 2 machine has been modified, although only limited independent evaluation of this is currently available.

The failure to include field testing in some surveys in developing countries is likely to lead to an underestimation of glaucoma prevalence. While reliance on the optic nerve appearance is not ideal, it would identify the more advanced cases and provide at least a minimum estimate of glaucoma prevalence. It would include those at highest risk for total blindness in their lifetimes.

This scheme is not definitive, but is intended as an operational approach to identifying, in cross sectional surveys, those suffering visual disability from glaucoma and to standardise our enumeration and evaluation of the cause of their disease. The usefulness of the proposed system for comparison between studies must now be validated by subsequent research.

\section{ACKNOWLEDGMENTS}

We thank all those mentioned in the appendix for participating in this work, and wish to emphasise that the views expressed in this manuscript are solely those of the authors, and not those of the ISGEO or an other organisation or individual.

\section{APPENDIX}

ISGEO Glaucoma classification working group, 27 and 28 June 1998, Leeuwenhorst, Netherlands: co-chair: Gordon J Johnson, Harry A Quigley; rapporteurs: Ralf Buhrmann, Paul J Foster; group members: Poul-Helge Alsbirk, Michelle Coffey, Lalit Dandona, Paulus TVM de Jong, Fridbert Jonasson, Paul Mitchell, Ian Murdoch, R Pararajasegaram, RS Ramrattan, Poul Roux, Ravi Thomas, Bjorn Thylefors, Roger Wolfs; co-opted advisers: Anders Heijl, David Henson, Roger A Hitchings, Chris A Johnson, Gottfried Naumann, John F Salmon; co-authors of studies from which data are presented: Bangladesh: M Rahman, N Rahman, AU Zahman, A Zia; Mongolia: J Bassanhu, J Devereux, D Uranchimeg, PS Lee, D Machin; Singapore: SKL Seah, F Oen, TP Ng, D Machin, J Devereux, J Hall, J Hee, SJ Chew, PT Khaw; Tanzania: Y Barron, SK West, MS Oliva, BBO Mmbaga.

\section{Authors' affiliations}

P J Foster, G J Johnson, Department of Epidemiology and International Eye Health, Institute of Ophthalmology, Bath Street, London ECIV 9EL, UK

R Buhrmann, University of Ottawa Eye Institute, Ottawa, Ontario, Canada

H A Quigley, Dana Center for Preventive Ophthalmology, Johns Hopkins University, Baltimore, Maryland, USA

\section{REFERENCES}

1 Buhrmann RR, Quigley HA, Barron Y, et al. Prevalence of glaucoma in a rural East African population. Invest Ophthalmol Vis Sci 2000;41:40-8.

2 Foster PJ, Baasanhu J, Alsbirk PH, et al. Glaucoma in Mongolia-a population-based survey in Hövsgöl Province, Northern Mongolia. Arch Ophthalmol 1996;114:1235-41.

3 Foster PJ, Oen FT, Machin DS, et al. The prevalence of glaucoma in Chinese residents of Singapore. A cross-sectional population survey in Tanjong Pagar district. Arch Ophthalmol 2000;1 18:1 105-11.

4 Quigley HA. Number of people with glaucoma worldwide. Br J Ophthalmol 1996;80:389-93.
5 Asman P, Heiil A. Diffuse visual field loss and glaucoma. Acta Ophthalmol Scand 1994;72:303-8.

6 Viswanathan AC, McNaught Al, Poinoosawmy D, et al. Severity and stability of glaucoma. Patient perception compared with objective measurement. Arch Ophthalmol 1999:117:450-4.

7 Salmon JF, Mermoud A, lvey A, et al. The prevalence of primary angle-closure glaucoma and open angle glaucoma in Mamre, Western Cape, South Africa. Arch Ophthalmol 1993;111:1263-9.

8 Congdon N, Quigley HA, Hung PT, et al. Screening techniques for angle-closure glaucoma in rural Taiwan. Acta Ophthalmol 1996;74:113-19

9 Douglas GR, Drance SM, Schulzer M. The visual field and nerve head in angle-closure glaucoma. A comparison of the effects of acute and chronic angle closure. Arch Ophthalmol 1975;93:409-1 1

10 Dhillon B, Chew PT, Lim ASM. Field loss in primary angle-closure glaucoma. Asia-Pacific J Ophthalmol 1990;2:85-7.

11 Jonas JB, Gusek GC, Guggenmoos-Holzmann I, et al. Size of the optic nerve scleral canal and comparison with intravital determination of optic disc dimensions. Graefes Arch Clin Exp Ophthalmol 1988;226:213-15.

12 Jonas JB, Gusek GC, Naumann GO. Optic disc, cup and neuroretinal rim size, configuration and correlations in normal eyes [published errata appear in Invest Ophthalmol Vis Sci 1991;32:1893 and 1992;32:474-5]. Invest Ophthalmol Vis Sci 1988;29:1 151-8.

13 Jonas JB, Muller-Bergh JA, Schlotzer-Schrehardt UM, et al. Histomorphometry of the human optic nerve. Invest Ophthalmol Vis Sci 1990;31:736-44.

14 Jonas JB, Gusek GC, Guggenmoos-Holzmann I, et al. Correlations of the neuroretinal rim area with ocular and general parameters in normal eyes. Ophthalmic Res 1988;20:298-303.

15 Jonas JB, Schmidt AM, Muller-Bergh JA, S et al. Human optic nerve fiber count and optic disc size. Invest Ophthalmol Vis Sci 1992;33:2012-18.

16 Montgomery DM. Clinical disc biometry in early glaucoma. Ophthalmology 1993;100:52-6.

17 Spencer AF, Vernon SA. Optic disc measurement with the Zeiss four mirror contact lens. Br J Ophthalmol 1994;78:775-80.

18 Garway-Heath DF, Ruben S, Viswanathan AC, et al. Vertical cup/disc ratio in relation to optic disc size: its value in the assessment of the glaucoma suspect. Br J Ophthalmol 1998;82:1118-24.

19 Varma R, Tielsch JM, Quigley HA, et al. Race-, age-, gender-, and refractive error-related differences in the normal optic disc. Arch Ophthalmol 1994;112:1068-76.

20 Beck RW, Messner DK, Musch DC, et al. Is there a racial difference in physiologic cup size? Ophthalmology 1985;92:873-6.

21 Chi T, Ritch R, Stickler D, et al. Racial differences in optic nerve head parameters. Arch Ophthalmol 1989;107:836-9.

22 Wolfs RC, Borger PH, Ramrattan RS, et al. Changing views on open-angle glaucoma: definitions and prevalences-The Rotterdam Study. Invest Ophthalmol Vis Sci 2000;41:3309-21.

23 Erie JC, Hodge DO, Gray DT. The incidence of primary angle-closure glaucoma in Olmstead County, Minnesota. Arch Ophthalmol 1997; 115:177-81.

24 Clemmesen V, Alsbirk PH. Primary angle-closure glaucoma (ACG) in Greenland. Acta Ophthalmol 1971;49:47-58.

25 Alsbirk PH. Anatomical risk factors in primary angle-closure glaucoma. A ten year follow up survey based on limbal and axial anterior chamber depths in a high risk population. Int Ophthalmol 1992;16:265-72.

26 Johnson CA, Cioffi GA, Drance SM, et al. A multicenter comparison study of the Humphrey field analyzer I and the Humphrey field analyzer II. Ophthalmology 1997:104:1910-17.

27 Bengtsson B, Heijl A, Olsson J. Evaluation of a new threshold visual field strategy, SITA, in normal subjects. Swedish Interactive Thresholding Algorithm. Acta Ophthalmol Scand 1998;76:165-9.

28 Henson D, Evans J, Chauhan BC, et al. Influence of fixation accuracy on threshold variability in patients with open angle glaucoma. Invest Ophthalmol Vis Sci 1996;37:444-50

29 Vingrys AJ, Demirel S. False-response monitoring during automated perimetry. Optom Vis Sci 1998;75:513-17.

30 Alsbirk PH. Anterior chamber depth and primary angle-closure glaucoma. I. An epidemiologic study in Greenland Eskimos. Acta Ophthalmol 1975;53:89-104.

31 Arkell SM, Lightman DA, Sommer A, et al. The prevalence of glaucoma among eskimos of Northwest Alaska. Arch Ophthalmol 1987; 105:482-5.

32 Hu Z, Zhao ZL, Dong FT. [An epidemiological investigation of glaucoma in Beijing and Shun-yi county.] [Chinese] Chung-Hua Yen Ko Tsa Chih [Chinese Journal of Ophthalmology] 1989;25:115-18.

33 Zhao JL. [An epidemiological survey of primary angle-closure glaucoma (PACG) in Tibet.] [Chinese] Chung-Hua Yen Ko Tsa Chih [Chinese Journal of Ophthalmology] 1990;26:47-50.

34 Shiose Y, Kitazawa Y, Tsukuhara S, et al. Epidemiology of glaucoma in Japan - a nationwide glaucoma survey. Jpn J Ophthalmol $1991 ; 35: 133-55$ 